\title{
Loss of alpha power is related to increased gamma synchronization A marker of reduced inhibition in tinnitus?
}

\section{Loss of alpha power is related to increased gamma synchronization-A marker of reduced inhibition in tinnitus?}

\author{
Isabel Lorenz*, Nadia Müller, Winfried Schlee, Thomas Hartmann, Nathan Weisz \\ University of Konstanz, Konstanz, Germany
}

\begin{abstract}
A B S T R A C T
Tinnitus is the perception of sound in the absence of any external auditory stimulus. Based on previous research we have proposed a framework which postulates that the reduction of ongoing inhibitory alpha activity in tinnitus subjects favors a synchronization of neurons in the gamma frequency range while in a resting state. In the present work we are validating the existence of an inverse relationship between auditory gamma and alpha activity in tinnitus and control subjects using Magnetoencephalography. Tinnitus subjects exhibited a significantly steeper slope of the regression line compared to controls, presumably because a greater number of subjects concurrently exhibited low alpha and high gamma power. Therefore, the role of the alpha-gamma pattern is discussed regarding its possible implication for the generation of tinnitus.
\end{abstract}

Tinnitus is the subjective perception of sound (e.g., a ringing or hissing noise) in the absence of any external stimulus. The condition affects approximately $5-15 \%$ of the population in western societies [6] and to date the underlying neuronal mechanisms of tinnitus are still not completely understood. One likely trigger of tinnitus is damage to the peripheral hearing system. This damage leads to aberrant central auditory activity, i.e. an overall increase in the firing of neurons, especially in the dorsal cochlear nucleus [12], the inferior colliculus, the primary and the secondary auditory cortex [6]. Furthermore, it has been shown that populations of neurons with an increased firing rate locally synchronize their activity [21].

In previous research using Magnetoencephalography (MEG), our group demonstrated abnormalities in the power spectrum of chronic tinnitus patients while in the resting state [32,33]. These changes were characterized by a noticeable reduction of power in the alpha $(8-12 \mathrm{~Hz})$ frequency band and a concomitant enhancement in the delta $(0.5-4 \mathrm{~Hz})$ as well as the gamma $(>30 \mathrm{~Hz})$ frequency range in temporal cortical regions. To further examine these abnormalities we are going to concentrate on the interaction of alpha and gamma power in the present work.

In recordings of spontaneous activity, alpha usually dominates the power spectrum since alpha rhythms can be found in virtually all sensory and motor areas while subjects are at

\footnotetext{
* Corresponding author at: Department of Psychology, University of Konstanz, P.O. Box D25, 78458 Konstanz, Germany. Tel.: +49 753188 3571;

fax: +497531884601

E-mail address: Isabel.Lorenz@uni-konstanz.de (I. Lorenz).
}

rest [2]. However, this does not mean that alpha rhythms are the neural code of 'doing nothing' as the frequently-used term 'idling-rhythm' may imply. Based on studies of event-related alpha reductions ('Event-Related Desynchronization'; ERD) and enhancements ('Event-Related Synchronization'; ERS) Klimesch et al. [15] hypothesized that oscillations in the alpha frequency range emerge from rhythmical fluctuations of inhibitory neurons. This notion finds support in recent studies which combine Electroencephalography (EEG) with Transcranial Magnetic Stimulation (TMS) [10,14,23].

Neuronal activity in the gamma frequency range, however, has been demonstrated to be associated with almost all categories of higher-order cognitive functions such as feature specification and cognitive binding $[22,28,9]$. Gamma activity has been associated with synchronization of firing within [8] as well as between neuronal cell-assemblies [27].

The existence of an antagonistic nature of alpha and gamma rhythms has been indicated, for example, by fMRI studies relating signal changes in blood oxygen level-dependent (BOLD) to changes in electrophysiological responses [18,16]: Gamma activity is positively correlated with the BOLD signal [18], whereas alpha activity exhibits a negative relationship [16].

From a clinical perspective, enhanced neuronal activity in the gamma frequency range in the resting state has been linked to positive symptoms in some neuropsychiatric disorders, including tinnitus [17].

With the Synchronization-by-Loss-of-Inhibition-Model (SLIM) [31] we recently proposed a framework to explain the observed oscillatory changes in tinnitus and to derive scientific as well as clinical predictions. This model essentially argues that input depri- 
vation (caused by deafferentation due to hearing loss) leads to reduced activity and therefore to an enhancement of delta power along the tonotopic axis. In addition to a suppression of excitatory neuron activity, the activity of inhibitory neurons, whose impact normally is reflected as alpha activity $[15,19,24]$, is also suppressed. The exact origin of alpha is still a matter of ongoing debate, yet recent findings [3] indicate that at least some forms of alpha emerge from the intracortical interactions of neurons, rather than just being passively driven by thalamic afferents. The assumed 'release of inhibition', may indicate a spontaneous synchronization of firing of excitatory neurons in circumscribed regions of the tonotopic axis. Increased synchronized neuronal activity is then reflected within the gamma frequency range. Since synchronized activity is crucial for a coherent percept, abnormally enhanced gamma activity in the auditory cortex might be a necessary requirement of the sound perception in tinnitus [33]. Chronification of tinnitus could result from longer lasting synchronous firing, leading to enhanced synaptic efficacy within a neuronal cell assembly via Hebbian mechanisms.

If dominant resting alpha activity reflects a mechanism that suppresses undesirable 'spontaneous' high-frequency synchronization, as postulated by SLIM [31], then one prediction, which has been untested to date, would be that levels of auditory alpha and gamma activity are inversely related. The purpose of the present MEG study was to test this hypothesis of an inverse relationship between alpha and gamma activity in tinnitus and control subjects. Furthermore, the influence of tinnitus on such a relationship is examined more closely.

Twenty-six subjects suffering from chronic tinnitus participated in the study ( 20 male, 6 female). The mean age was 45 years (range: $20-65$ years); the mean tinnitus duration 6.75 years $( \pm 1.01)$. Subjects were recruited via advertisements in the local newspaper and flyers at the University of Konstanz. Nine subjects reported dominant left, 10 bilaterally equal, and 7 dominant right lateralized tinnitus. Tinnitus distress was assessed using the German version of the Tinnitus Questionnaire [7]. The average distress score was 27.48 ( \pm 3.54$)$, corresponding to mild distress. Twenty-one subjects without tinnitus or other hearing disorder served as a control group ( 16 males, 5 females, mean age: 35 years; range: $23-78$ years). None of the subjects has participated in an MEG study before. The experiment has been conducted in accordance with the declaration of Helsinki and with adequate understanding and written informed consent of the subjects.

Five minutes of MEG under a resting condition were recorded (sampling rate: $678.17,0.1-200 \mathrm{~Hz}$ band-pass) using a 148-channel whole-head magnetometer (Magnes $2500 \mathrm{WH}$; 4-D Neuroimaging, San Diego, CA) in a magnetically shielded chamber (Vakuumschmelze, Hanau, Germany). Participants lay in a supine position and were requested to keep eyes open and to focus on a fixed point on the ceiling. Four electrodes were used to record eye movements (electrooculogramm, EOG): two electrodes were positioned at the left and right outer canthi (horizontal EOG) and two were placed above and below the right eye. Additionally, electrocardiogram was recorded from two electrodes on the left and right forearm. For the MEG measurement the head position within the MEG helmet had to be assessed, thus positions of five index points and individual head shapes were sampled using a digitizer.

Data were corrected for eye and heartbeat-related artifacts using a multiple source approach [1] implemented in BESA (MEGIS Software, Gräfeling, Germany). Afterwards the data were projected from the 148 sensors onto a source montage consisting of eight regional sources analogously to our previous work [34]. A source model was generated using so-called regional sources consisting of two orthogonal dipoles (the radial source is discarded) in order to capture global activity independent of the orientation. Two of the eight sources were placed symmetrically and bilaterally in the audi-

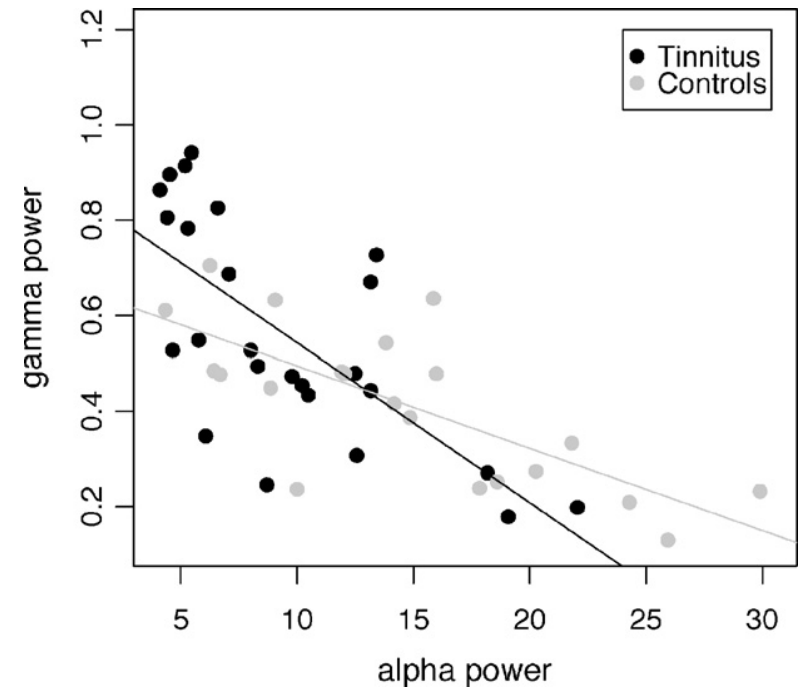

Fig. 1. Correlation between temporal alpha and gamma power averaged across both temporal sources. Black dots indicate values of tinnitus subjects; grey dots display values of controls.

tory cortex, which was the region of interest. The other six sources were distributed over the cortex: left and right prefrontal cortex, left and right parietal cortex, anterior cingulum, and occipital cortex. The locations of sources were predefined by the authors, however, and should therefore not be confused with the more precise activity localizations drawn from functional neuroimaging studies. The strength of applying a source montage is that the activity of brain regions of interest (i.e. left and right auditory cortex) can be captured with a considerably reduced contribution of other concurrently active brain regions. Hence, the other six sources acted as a kind of spatial filter, sharpening the activity from the regions of interest. The location of sources was identical for all subjects (adapted to the radius of the single-sphere fitted to the headshape of the individual) and covered roughly the whole volume (for detailed information concerning source montages see [25]). The leadfield was computed for the regions of interest and its pseudo-inverse was used for calculation of the source strength. Data were then downsampled to $450 \mathrm{~Hz}$ and exported to Matlab (The MathWorks, Natick, MA) for further analysis. For data analysis the entire 5 min length were used, analogously to our previous work $[32,33]$. The powerspectra for each source was averaged across orientations to yield a global estimate of the energy at that specific brain region. There was no difference in the global power between tinnitus and control subjects. Normalized power was computed to minimize variance resulting from strong interindividual variability. Linear regressions were calculated for the left and the right hemisphere between alpha (8-12) and gamma $(40-90 \mathrm{~Hz}$; excluding $49-51 \mathrm{~Hz})$ power. Additionally, group membership was considered as a factor in order to uncover potential differences in regression slopes. The significance level was set at $5 \%$.

A strong negative correlation between temporal alpha and gamma power existed for tinnitus subjects $(r=-.70, p<.0001)$ as well as for controls $(r=-.74, p<.001)$. These results are averaged across both temporal sources (Fig. 1). Results for each side, respectively, are for tinnitus subjects left temporally $(r=-.72, p<.0001)$, right temporally $(r=-.63, p<.001)$ and for control subjects left temporally $(r=-.79, p<.0001)$, right temporally $(r=-.65, p<.01)$. Furthermore, the steepness of the regression lines for tinnitus and control subjects was compared to examine differences in the alpha-gamma relationship between tinnitus and control subjects. A significantly steeper slope was revealed for the tinnitus subjects $(F=4.48, p<.05)$ averaged across both temporal sources (left 
temporally separately: $F=4.22, p=.05$, right temporally separately: $F=3.82, p=.06$ ).

In the present work we demonstrated the existence of an inverse relationship between auditory gamma and alpha activity in resting tinnitus as well as in control subjects using MEG. This finding is in line with a prediction derived from the SLIM model outlined in the introduction. The steepness of the regression lines was compared for both groups to examine differences between tinnitus and control subjects regarding the alpha-gamma pattern. Tinnitus subjects demonstrated a significantly steeper slope of the regression line compared to controls, putatively caused by the fact that a greater number of tinnitus subjects concurrently exhibit low alpha and high gamma power (Fig. 1). However, Fig. 1 clearly depicts a strong overlap between tinnitus and control subjects and shows that - despite being statistically significant - the group difference in slope is quite small. Hence, the question arises why some subjects experience tinnitus while others with a similar alpha-gamma pattern do not. Different explanations are conceivable for this phenomenon and would require further investigation: (1) alpha does not solely reflect the impact of an ongoing inhibition, but a complex mixture of several activities. Viewed from a macroscopic level it would not be possible to discern the various mechanisms for resting data. (2) As suggested by SLIM, the combination of enhanced slow-waves (most likely due to deafferentation) combined with a reduction of alpha-power (suggested to be inhibitory) leads to enhanced gamma synchronization. Since there is no increase of slow-wave power in the healthy control subjects, a reduction of alpha does not lead to an increase in gamma. (3) A continuously enhanced level of synchronized activity (via reduced inhibition) within the auditory cortex enhances the baseline sensitivity for neurons to synchronize via neuroplastic processes (e.g. enhanced synaptic strength), leading to a stabilization of the tinnitus-perception related activity. (4) The degree of synchronization/inhibition within the auditory cortex is influenced by the dynamics in a widespread neuronal network exceeding the auditory cortex. Thus, whether one is experiencing a conscious percept such as tinnitus depends strongly on how auditory cortical regions are likely to affect downstream higher-order brain regions. This could be achieved, e.g. by a higher output of auditory cortical regions (e.g. reflected in gamma activity) and/or a higher sensitivity of downstream brain regions to process the input from auditory regions. In order to examine this issue further, studies investigating the connectivity pattern between brain regions in tinnitus as compared to controls will be highly informative. Some evidence for this explanation has recently been found in a study by our group which revealed a functional cortical architecture with altered inter-areal synchronization in the alpha and gamma frequencies in tinnitus subjects [26]. Low inter-areal synchronization in the alpha network - as in the case of tinnitus - is accompanied by high synchronization in the gamma network. (5) A further phenomenon potentially influencing the current results, which has been unaccounted for, are the individual differences between tinnitus subjects. These subjects may differ according to their hearing loss, their tinnitus duration or the quality of their tinnitus (pure tone or white noise tinnitus) just to name a few. To our knowledge, there is no work correlating these factors to abnormal neuronal oscillations. There is, however, evidence from different TMS therapy studies that the amount of hearing loss, the tinnitus duration and the quality of the tinnitus seem to play an important role for the treatment outcome $[13,4]$. (6) Our data only reflect a 'snapshot' of cortical activity within a single subject. Therefore, not only the interindividual, but also the intraindividual variability comes into play. A steeper slope in the alpha-gamma relationship could indicate an altered gain within tinnitus sufferers, which is also suggested by evoked auditory data [35,5]. As the level of inhibitory drive is downregulated in tinnitus, neurons in the auditory cortex synchronize in a disproportionate manner. Thus, applied to spontaneous data, repeated measurements within one subject would need to be carefully investigated.

Overall, despite confirming the notion that alpha is crucial in regulating cortical excitability as well as the synchronization within cell assemblies, our results indicate that a dysfunction of the mechanism underlying alpha could be a necessary but not a sufficient prerequisite for the development of tinnitus.

A further question regarding our outcome is that the effect is clearly more pronounced within the left temporal source, whereas in the right temporal source only a trend could be revealed. These results may be traced back to lateralization effects of the tinnitus. Nevertheless, this topic certainly requires further research.

We did not find a linear correlation between changes in oscillatory power and tinnitus symptoms (distress measured with the Tinnitus Questionnaire [7]). It should be noted, however, that the subjective distress level in tinnitus is not correlated to psychoacoustic measures $[11,20,30]$. Hence, we consider alpha reductions and gamma enhancements in auditory brain regions not to be influenced by the distress level related to the tinnitus. Nevertheless, it would be interesting in future to employ adequate psychoacoustic measures of tinnitus in order to investigate whether the abnormal oscillatory patterns are related to basic psychoacoustical features of the tinnitus sensation.

Another aspect to be taken into consideration regarding lower alpha power in tinnitus subjects is that these subjects may have had a higher general arousal during the measurement due to their knowledge that potential causes of their discomforting ear noise will be examined. This may have resulted in lower alpha power. However, we consider it to be highly unlikely that this has caused a significant group effect regarding the slopes of the alpha and gamma relationship. Furthermore, we have examined auditory alpha and gamma power, whereas arousal/relaxation would rather be reflected in decreased/enhanced posterior alpha power [29]. Furthermore, the fact that the overall distress level is not related to alpha or gamma power is an indicator for the effects not to be resulting from arousal differences (assuming that distressed participants should be more aroused).

Within our framework, which was delineated in the introduction (SLIM) [31], our current findings support the notion that reduced ongoing inhibition is associated with enhanced gamma synchronization in tinnitus and in control subjects. Our results are in line with previous research conducted by our group which demonstrated aberrant patterns of spontaneous neuronal activity in tinnitus subjects, namely reduced alpha and enhanced delta and gamma power [32]. Since alpha has been described as an inhibitory mechanism for the synchronization of power in higher frequencies $[15,24,23]$, the strong reduction of alpha power in tinnitus subjects probably increases the likelihood of 'spontaneous synchronization, macroscopically reflected in increased gamma power. As mentioned above, however, this local type of synchronization is not sufficient to fully explain the conscious perception of the tinnitus sound because it will also have to be taken into account to what extent these processes affect downstream (higher-order) brain regions. This is an important issue when further developing the SLIM model.

In conclusion, the present MEG study provides support for an inverse relationship between auditory alpha and gamma band activity in resting spontaneous neuronal activity. This finding provides direct support for our model (SLIM) inasmuch as an interruption of the inhibitory function of the alpha rhythm is associated with synchronization in the gamma frequency range. With these novel findings regarding the strong negative association between gamma and alpha band activity in general, further - more targeted - investigations (e.g., in animals) concerning the alpha-gamma pattern in tinnitus would be important. 


\section{Acknowledgements}

This research was supported by the Deutsche Forschungsgemeinschaft (DFG) and the Tinnitus Research Initiative (TRI). The authors would like to thank Simona Müller for help during data acquisition and Christian Lorenz for helpful comments on a draft of this article. We would also like to thank Christina Robert for proofreading this manuscript.

\section{References}

[1] P. Berg, M. Scherg, A multiple source approach to the correction of eye artifacts, Electroencephalogr. Clin. Neurophysiol. 90 (1994) 229-241.

[2] H. Berger, Über das Electroencephalogramm des Menschen, Arch. Psychiat. Nervenkr. 87 (1929) 527-570.

[3] A. Bollimunta, Y. Chen, C.E. Schroeder, M. Ding, Neuronal mechanisms of cortical alpha oscillations in awake-behaving macaques, J. Neurosci. 28 (2008) 9976-9988.

[4] D. De Ridder, E. van der Loo, K. Van der Kelen, T. Menovsky, P. van de Heyning, A. Moller, Do tonic and burst TMS modulate the lemniscal and extralemniscal system differentially? Int. J. Med. Sci. 4 (2007) 242-246.

[5] E. Diesch, M. Struve, A. Rupp, S. Ritter, M. Hulse, H. Flor, Enhancement of steadystate auditory evoked magnetic fields in tinnitus, Eur. J. Neurosci. 19 (2004) 1093-1104.

[6] J.J. Eggermont, L.E. Roberts, The neuroscience of tinnitus, Trends Neurosci. 27 (2004) 676-682.

[7] G. Goebel, W. Hiller, Tinnitus Fragebogen (TF): Ein Instrument zur Erfassung von Belastung und Schweregrad bei Tinnitus, 1998.

[8] C.M. Gray, P. Konig, A.K. Engel, W. Singer, Oscillatory responses in cat visua cortex exhibit inter-columnar synchronization which reflects global stimulus properties, Nature 338 (1989) 334-337.

[9] R. Hannemann, J. Obleser, C. Eulitz, Top-down knowledge supports the retrieval of lexical information from degraded speech, Brain Res. 1153 (2007) 134-143.

[10] F. Hummel, F. Andres, E. Altenmuller, J. Dichgans, C. Gerloff, Inhibitory control of acquired motor programmes in the human brain, Brain 125 (2002) 404420.

[11] S.C. Jakes, R.S. Hallam, C. Chambers, R. Hinchcliffe, A factor analytical study of tinnitus complaint behaviour, Audiology 24 (1985) 195-206.

[12] J.A. Kaltenbach, The dorsal cochlear nucleus as a participant in the auditory, attentional and emotional components of tinnitus, Hear Res. 216-217 (2006) 224-234.

[13] T. Kleinjung, T. Steffens, P. Sand, T. Murthum, G. Hajak, J. Strutz, B. Langguth, P. Eichhammer, Which tinnitus patients benefit from transcranial magnetic stimulation? Otolaryngol. Head Neck Surg. 137 (2007) 589-595.

[14] W. Klimesch, P. Sauseng, C. Gerloff, Enhancing cognitive performance with repetitive transcranial magnetic stimulation at human individual alpha frequency, Eur. J. Neurosci. 17 (2003) 1129-1133.

[15] W. Klimesch, P. Sauseng, S. Hanslmayr, EEG alpha oscillations: the inhibitiontiming hypothesis, Brain Res. Brain Res. Rev. 53 (2007) 63-88.
[16] H. Laufs, A. Kleinschmidt, A. Beyerle, E. Eger, A. Salek-Haddadi, C. Preibisch, K. Krakow, EEG-correlated fMRI of human alpha activity, Neuroimage 19 (2003) 1463-1476.

[17] R.R. Llinás, U. Ribary, D. Jeanmonod, E. Kronberg, P.P. Mitra, Thalamocortica dysrhythmia: A neurological and neuropsychiatric syndrome characterized by magnetoencephalography, in: Proceedings of the National Academy of Sciences of the United States of America, vol. 96, 1999, pp. 15222-15227.

[18] N.K. Logothetis, J. Pauls, M. Augath, T. Trinath, A. Oeltermann, Neurophysiological investigation of the basis of the fMRI signal, Nature 412 (2001) 150-157.

[19] B.K. Min, C.S. Herrmann, Prestimulus, EEG alpha activity reflects prestimulus top-down processing, Neurosci. Lett. 422 (2007) 131-135.

[20] C.W. Newman, J.A. Wharton, G.P. Jacobson, Self-focused and somatic attention in patients with tinnitus, J. Am. Acad. Audiol. 8 (1997) 143-149.

[21] A. Norena, C. Micheyl, S. Chery-Croze, L. Collet, Psychoacoustic characterization of the tinnitus spectrum: implications for the underlying mechanisms of tinnitus, Audiol. Neurootol. 7 (2002) 358-369.

[22] F. Pulvermuller, A. Keil, T. Elbert, High-frequency brain activity: perception or active memory? Trends Cogn. Sci. 3 (1999) 250-252.

[23] T.A. Rihs, C.M. Michel, G. Thut, Mechanisms of selective inhibition in visual spatial attention are indexed by alpha-band EEG synchronization, Eur. J. Neurosci. 25 (2007) 603-610.

24] V. Romei, T. Rihs, V. Brodbeck, G. Thut, Resting electroencephalogram alpha-power over posterior sites indexes baseline visual cortex excitability, Neuroreport 19 (2008) 203-208.

[25] M. Scherg, N. Ille, H. Bornfleth, P. Berg, Advanced tools for digital EEG review: virtual source montages, whole-head mapping, correlation, and phase analysis, J. Clin. Neurophysiol. 19 (2002) 91-112.

[26] W. Schlee, K. Dohrmann, T. Hartmann, I. Lorenz, N. Müller, T. Elbert, N. Weisz, Assessment and modification of the tinnitus-related cortical network, Semin. Hearing 29 (2008) 270-287, Thieme.

[27] W. Singer, Neuronal synchrony: a versatile code for the definition of relations? Neuron 24 (1999) 49-65, 111-125.

[28] C. Tallon-Baudry, O. Bertrand, Oscillatory gamma activity in humans and its role in object representation, Trends Cogn. Sci. 3 (1999) 151-162.

[29] H. van Dijk, J.M. Schoffelen, R. Oostenveld, O. Jensen, Prestimulus oscillatory activity in the alpha band predicts visual discrimination ability, J. Neurosci. 28 (2008) 1816-1823.

[30] E.D. van Veen, J.B. Jacobs, J.M. Bensing, Assessment of distress associated with tinnitus, J. Laryngol. Otol. 112 (1998) 258-263.

[31] N. Weisz, K. Dohrmann, T. Elbert, The relevance of spontaneous activity for the coding of the tinnitus sensation, Prog. Brain Res. 166 (2007) 61-70.

[32] N. Weisz, S. Moratti, M. Meinzer, K. Dohrmann, T. Elbert, Tinnitus perception and distress is related to abnormal spontaneous brain activity as measured by magnetoencephalography, PLoS Med. 2 (2005) e153.

[33] N. Weisz, S. Müller, W. Schlee, K. Dohrmann, T. Hartmann, T. Elbert, The neural code of auditory phantom perception, J. Neurosci. 27 (2007) 1479-1484.

[34] N. Weisz, C. Wienbruch, K. Dohrmann, T. Elbert, Neuromagnetic indicators of auditory cortical reorganization of tinnitus, Brain 128 (2005) 2722-2731.

[35] C. Wienbruch, I. Paul, N. Weisz, T. Elbert, L.E. Roberts, Frequency organization of the $40-\mathrm{Hz}$ auditory steady-state response in normal hearing and in tinnitus, Neuroimage 33 (2006) 180-194. 